\title{
A spectroscopic study of the $\beta$ Cephei star 12 (DD) Lacertae
}

\author{
M. Desmet, ${ }^{1}$ M. Briquet, ${ }^{1}$ P. De Cat, ${ }^{2}$ C. Aerts,,${ }^{1,3}$ G. Handler, ${ }^{4}$ J. Krzesinski, ${ }^{5}$ \\ H. Lehmann, ${ }^{6}$ S. Masuda, ${ }^{7}$ P. Mathias, ${ }^{8}$ D. E. Mkrtichian, ${ }^{9}$ J. H. Telting, ${ }^{10}$ \\ K. Uytterhoeven, ${ }^{11}$ S. L. S. Yang ${ }^{12}$ \\ ${ }^{1}$ Institute of Astronomy - KULeuven, Celestijnenlaan 200D, 3001 Leuven, Belgium \\ ${ }^{2}$ Koninklijke Sterrenwacht van België, Ringlaan 3, 1180 Brussel, Belgium \\ ${ }^{3}$ Department of Astrophysics, University of Nijmegen, PO Box 9010, Nijmegen, The Netherlands \\ ${ }^{4}$ Institut für Astronomie, Universität Wien, 1180 Wien, Austria \\ ${ }^{5}$ Mt. Suhora Observatory, Cracow Pedagogical University, UI. Podchorazych 2, 30-084 Cracow, Poland \\ ${ }^{6}$ Karl-Schwarzschild-Observatorium, Thüringer Landessternwarte, 7778 Tautenburg, Germany \\ ${ }^{7}$ Okayama Astrophysical Obs., National Astronomical Obs., Kamogata, Okayama, Japan \\ ${ }^{8}$ Observatoire de la Côte d'Azur, Dpt. Gemini, UMR 6203, 06103 Grasse, France \\ ${ }^{9}$ Astronomical Observatory of Odessa National University, Marazlievskaya 1v, 65014 Odessa, Ukraine \\ ${ }^{10}$ Nordic Optical Telescope, Apartado 474, 38700 Santa Cruz de La Palma \\ ${ }^{11}$ Brera Astronomical Observatory, Via E. Bianchi 46, 23807 Merate (LC), Italy \\ 12 Department of Physics and Astronomy, University of Victoria, Victoria, BC V8W 3P6, Canada
}

\begin{abstract}
We present first results of a spectroscopic multisite campaign for 12 (DD) Lacertae (hereinafter $12 \mathrm{Lac}$ ). This star is one of the best observed $\beta$ Cephei stars. It has a large number of known oscillation frequencies but a lack of identified $\mathrm{m}$-values for its detected modes. In our data set we find seven independent frequencies together with combination frequencies. In addition, two of these modes are identified: one radial mode and one prograde dipole mode.
\end{abstract}

\section{Data}

The data originate from a spectroscopic multisite campaign for 12 Lac. We also added the data set from Mathias et al. (1994). Almost two thousand high-resolution spectra were gathered with 8 different telescopes, which were evenly spread over the northern continents to reduce aliasing of the frequencies. All spectra include the SiIII triplet centred on $4560 \AA$ and were collected during a time span of 12 years, from September 1990 until July 2004.

\section{Frequency analysis}

The SiII line profiles around $4560 \AA$ were subjected to a detailed line profile analysis. We computed the first three normalized velocity moments $\langle v\rangle,\left\langle v^{2}\right\rangle$ and $\left\langle v^{3}\right\rangle$ (for a definition see Aerts, De Pauw \& Waelkens 1992). The summation limits were determined dynamically for each individual line profile to avoid the noisy continuum. We applied Period04 (Lenz \& Breger 2005) to search for frequencies in $\langle v\rangle$, which is the centroid velocity. We also performed a two-dimensional frequency search (across the line profile) in order to find additional frequencies. In total we found seven independent frequencies, together with some combination frequencies. We clearly recovered the five main frequencies which had already been discovered spectroscopically in the past (e.g., Mathias et al. 1994). Even without the inclusion of the data set by Mathias et al. (1994), these frequencies are present. We also found two other additional independent frequencies, which have also been detected in photometric data by Handler et al. (2006): $0.35529 \pm 0.00001 \mathrm{~cd}^{-1}\left(1.8 \pm 0.1 \mathrm{~km} \mathrm{~s}^{-1}\right.$ in $\left.\langle v\rangle\right)$ and $7.40637 \pm 0.00001 \mathrm{~cd}^{-1}\left(0.6 \pm 0.1 \mathrm{~km} \mathrm{~s}^{-1}\right.$ in $\left.\langle v\rangle\right)$. The peaks in the periodogram corresponding to these two frequencies exceed an amplitude signal-to-noise ratio of 4 and thus they are significant (e.g., Breger et al. 1999). 


\section{Mode identification}

The main strength of mode identification techniques based on high-resolution spectroscopy is that they are able to derive the azimuthal order $m$ of the pulsation modes. Therefore, we adopt the values for the spherical degree $\ell$ of the pulsation modes provided by unique identifications based on multi-colour photometry (Handler et al. 2006) and use our data to derive $\mathrm{m}$. This technique has already been proven successful in the case of $\theta$ Ophiuchi (Briquet et al. 2005). Our conclusions are based on three different spectroscopic identification techniques. As a first result, we showed that one of the frequencies $\left(5.334229 \pm 0.000004 \mathrm{~cd}^{-1}, 2.8 \pm 0.1 \mathrm{~km} \mathrm{~s}^{-1}\right.$ in $\langle v\rangle)$ must be a radial or a dipole mode by means of the amplitude and phase variation across the line profile (Telting \& Schrijvers 1997, Schrijvers et al. 1997). This corroborates the identification of Handler et al. (2006), who concluded that this frequency corresponds to a radial mode. Secondly, to identify as many modes as possible, we are currently applying two additional state-of-the-art methods, namely the moment method (Briquet \& Aerts 2003) and the Fourier parameter fit (FPF) method (Zima 2006). In the moment method, the wavenumbers $(\ell, \mathrm{m})$ and some other continuous velocity parameters are computed in such a way that the theoretically computed first three moment variations best fit the observed ones. In the FPF method, the mode identification is performed by a $\chi^{2}$ minimization, using the observed zero point, amplitude and phase across the line profile and their theoretically modelled counterparts. Our preliminary results, based on the moment method and the FPF method, show that the frequency with the largest amplitude in the first moment $(5.178960 \pm$ $\left.0.000001 \mathrm{~cd}^{-1}, 14.3 \pm 0.1 \mathrm{~km} \mathrm{~s}^{-1}\right)$ corresponds to a prograde dipole mode $((\ell, m)=(1,1))$.

\section{Future work}

The ultimate goal is to construct stellar models which show oscillations in accordance with all the observed modes of $12 \mathrm{Lac}$, and thus constrain unknown stellar parameters. To this end, we will calculate an extensive grid of stellar models using the evolutionary code CLES (Code Liégeois d'Evolution Stellaire, written by R. Scuflaire).

Acknowledgments. MD, MB and CA are supported by the Research Fund, K.U. Leuven under the Grant GOA/2003/04. MB is Postdoctoral Fellow of the Fund for Scientific Research, Flanders.

\section{References}

Aerts C., De Pauw M., Waelkens C., 1992, A\&A, 266, 294

Breger M., Garrido R., Handler G., et al., 1999, A\&A, 349, 225

Briquet M., Aerts C., 2003, A\&A, 398, 687

Briquet M., Lefever K., Uytterhoeven K., Aerts C., 2005, MNRAS, 362, 619

Handler G., Jerzykiewicz M., Rodríguez E., et al., 2006, MNRAS, 365, 327

Lenz P., Breger M., 2005, Comm. Asteroseis., 146, 53

Mathias P., Aerts C., Gillet D., Waelkens C., 1994, A\&A, 289, 875

Schrijvers C., Telting J. H., Aerts C., Ruymaekers E., Henrichs H. F., 1997, A\&AS, 121, 343

Telting J. H., Schrijvers C., 1997, A\&A, 317, 723

Zima W., 2006, A\&A, 455, 227 